Meta

Journal des tradlucteurs

Translators' Journal

\title{
Fertilisation ou intégration
}

\section{Abdelaziz Mdibeh}

Volume 32, numéro 3, septembre 1987

La fertilisation terminologique dans les langues romanes

URI : https://id.erudit.org/iderudit/003609ar

DOI : https://doi.org/10.7202/003609ar

Aller au sommaire du numéro

Éditeur(s)

Les Presses de l'Université de Montréal

ISSN

0026-0452 (imprimé)

1492-1421 (numérique)

Découvrir la revue

Citer cet article

Mdibeh, A. (1987). Fertilisation ou intégration. Meta, 32(3), 278-284.

https://doi.org/10.7202/003609ar

Ce document est protégé par la loi sur le droit d'auteur. L'utilisation des services d'Érudit (y compris la reproduction) est assujettie à sa politique d'utilisation que vous pouvez consulter en ligne.

https://apropos.erudit.org/fr/usagers/politique-dutilisation/
Cet article est diffusé et préservé par Érudit.

Érudit est un consortium interuniversitaire sans but lucratif composé de l’Université de Montréal, l'Université Laval et l'Université du Québec à Montréal. Il a pour mission la promotion et la valorisation de la recherche. https://www.erudit.org/fr/ 


\section{FERTILISATION OU INTÉGRATION}

ABDELAZIZ MDIBEH

Faculté des lettres, Fès, Maroc

\section{1) HISTORIQUE}

Selon Meillet : "...les changements de structure sociale se traduisent par des changements de structure linguistique ${ }^{1} »$. Avant 1912 le Maroc avait une structure sociale certainement différente de celle qu'il allait commencer à connaître après le Protectorat en 1912.

Sa structure socio-économique avant 1912 était fondée sur l'agriculture dans les campagnes et l'artisanat dans quelques villes. L'événement du Protectorat en lui-même fut capital. Il détermina un changement de structure sociale et par suite un changement de structure linguistique. Car le nouvel arrivant a une langue, une culture et une économie différentes de celles du Maroc. Il fallait donc s'attendre à un bouleversement dans tous les domaines.

Le Protectorat avait installé une administration française et opté pour une économie moderne. Il avait fondé des écoles et des particuliers de la métropole avaient ouvert des usines, des magasins, des garages, des bars, etc.

Il en est donc résulté un contact entre autochtones et ressortissants français. Le contact entre individus de langues différentes crée des besoins de communication. Mais dans quelle langue fallait-il communiquer ? Bien entendu dans celle du pays qui dominait (voir L.-J. Calvet) ${ }^{2}$.

Et nous voilà donc dans une nouvelle structure socio-économique qui allait provoquer l'apprentissage d'une langue étrangère. Or, tout apprentissage d'une langue étrangère suppose un contact entre les langues en présence. Dans notre cas le contact a eu lieu entre l'arabe, le berbère d'une part, et le français d'autre part. (Dans cette intervention je ne m'occuperai que de l'arabe et du français.) Ce rappel historique était nécessaire pour montrer que les contacts de l'ADM ont eu lieu essentiellement avec le français en 1912 et après (ADM = arabe dialectal marocain).

De ce contact allait naître un phénomène multiséculaire : l'emprunt. D'ailleurs, qui dit emprunt dit " fertilisation terminologique " : il y a une langue qui emprunte à une autre langue. Cette "fertilisation terminologique » a surtout opéré au niveau lexical au Maroc. Mais la question qui se pose soixante-treize ans après ce premier contact massif est la suivante : y a-t-il «fertilisation terminologique » de l'ADM par le français ? Pour répondre à cette question, il faut effectuer des enquêtes lexicales dans tous les secteurs qui ont été envahis par les termes étrangers et particulièrement par les termes français. Cette tâche serait immense et demanderait le concours de plusieurs équipes de chercheurs et un organisme qui assurerait une coordination entre elles.

Pour tester cette question, j'ai dû me contenter d'une espèce de mini-enquête que j'ai effectuée sur le champ lexical du garage. En effet toute la terminologie du garage a été importée, puisque les premiers garagistes au Maroc étaient Français.

\section{2) L'ENQUÊTE}

Le garage où j'ai mené l'enquête dispose d'un outillage assez ancien. Le garagiste a suivi, il y a longtemps, des études dans une école professionnelle en langue française. 
Ceci m'a poussé à prendre comme informateurs ses 4 apprentis car ils connaissent bien les noms d'outils mais n'ont qu'une connaissance orale très rudimentaire du français. Ils sont donc moins affectés par le phonétisme et la morphosyntaxe du français que leur patron. C'est la raison pour laquelle je les ai choisis.

\section{FERTILISATION PHONOLOGIQUE?}

A. Analyse du corpus

1. Les voyelles

a) voyelles orales françaises

- $[\mathrm{e}]-[\varepsilon]$ et $[\mathrm{y}]$ se prononcent toutes $i$ en ADM

$[\mathrm{e}]>\mathrm{i}$ kle $>\mathrm{kli}$

$[\varepsilon]>\mathrm{i}$

$[y]>\mathrm{i}$

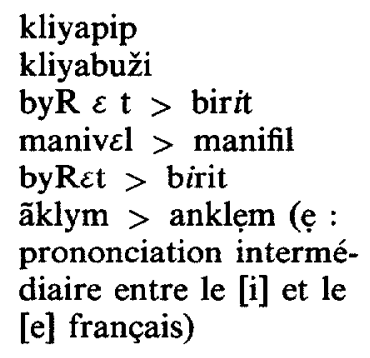

[e] français)

- Le [o] est prononcé $\bar{u}:$ fos $>$ lafũ ; žož $>$ lažǔž

- Le [ø] est prononcé $o:$ mwajø > mwayyo

- $[\Theta] 0:$ turnevis $>$ tornofis

- $[\mathrm{u}]>0:$ turnevis $>$ tornọfis

b) les voyelles nasales françaises

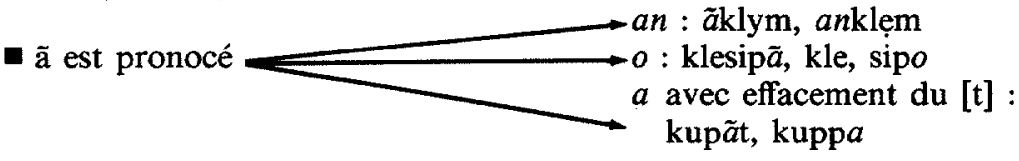

- $\tilde{\varepsilon}$ est prononcé an : pẽs > pans

2. Les consonnes
- $[1]>\mathrm{n}$
- $[\mathrm{R}]>1$
- $[\check{s}]>\check{z}$
- $[v]>\mathrm{f}$ ou $\mathrm{v}$
- $[\mathrm{p}]>\mathrm{p}$ ou $\mathrm{b}$
- $[\mathrm{t}]>\mathrm{t}$
kleamolet $>$ klamonet
Rəsor $>$ rəsol
kleafurš > sarut a fọrž
manivel $>$ manifil ou manivil
pẽs $>$ pans ou bans
ce $[t]$ n'est ni un $t$ ni un $t$ ( $t$ emphatique)

\section{D'autres phénomènes}

Nos informateurs ont recours à d'autres procédés phonétiques pour prononcer certains mots d'emprunts. 
a) La syncope

Ce procédé est un " phénomène très fréquent de disparition d'un ou plusieurs phonèmes à l'intérieur d'un $\operatorname{mot}^{3}$ ». kleamolct $>$ klamonẹt kle $>\mathrm{kl}$

b) L'épenthèse

«On appelle épenthèse le phénomène qui consiste à intercaler dans un mot ou un groupe de mots un phonème non étymologique pour des raisons d'euphonie, de commodité articulaire, par analogie...4

Ce phénomène que nous avons rencontré dans notre corpus consiste en un yod épenthétique (y).

$$
\begin{aligned}
& \text { kleabuži > kliyabuži } \\
& \text { kleadwil > kliyadwil } \\
& \text { kleapip > kliyapip }
\end{aligned}
$$

c) L'agglutination de l'article défini français

(i) termes commençant par une consonne au nom arabe : labirit (byRet) - lafūs (fosse) - lalim (lime) - lažǔž (jauge) - lanklem (enclume)

(ii) terme commençant par une voyelle : eto (étau) > lleto

Ce terme m'a poussé à me poser la question suivante : pourquoi les informateurs prononcent-ils ce terme avec un géminé à l'initiale. J'ai cherché d'autres termes en dehors du vocabulaire du garage et j'ai pu trouvé l'explication suivante qui est quasi générale.

$$
\begin{array}{lll}
\text { F } & \text { ADM } \\
\text { L'évier } & > & \text { llevye } \\
\text { l'école }> & \text { llekol } \\
\text { l'auto }> & \text { lloțo }
\end{array}
$$

En effet les termes qui commencent par une voyelle et qui reçoivent l'article défini français, sont prononcés avec un géminé en arabe à l'initiale. Je pense - et c'est une hypothèse qui reste à vérifier - que le $1^{\mathrm{er}} 1$ est l'article défini arabe et que le $2^{\mathrm{e}} 1$ est l'article défini français.

d) Prononciation mixte ou mélange

(i) le $1^{\text {er }}$ terme est traduit et prononcé en ADM

Cette prononciation consiste à prendre un terme d'emprunt construit (ici une synapsie), à traduire le $1^{\text {er }}$ terme et à le prononcer en français et à garder une prononciation française altérée pour le ou les autres termes - kleplat > sarut plat - klesipã $>$ sarut sipo - kle a furš > sarut a foř̌

(ii) Le $1^{\text {er }}$ terme et le joncteur sont traduits et prononcés en ADM

Le $2^{e}$ terme a une prononciation française altérée :

$$
\begin{array}{llll}
\text { sarut } & \text { dyal } & \text { lkilas } & \text { (clé pour culasse) } \\
\text { sarut } & \text { dəl } & \text { filtər } &
\end{array}
$$

\section{Conclusion sur ce paragraphe}

Après la description du corpus, essayons de répondre à la question posée au début de ce paragraphe. Y a-t-il eu intégration des termes d'emprunt dans le système phonologique de l'ADM ? Y a-t-il eu fertilisation phonologique de l'ADM par le français?

1. Les voyelles

Nous savons que les voyelles nasales $[\tilde{a}-\tilde{\mathbf{\jmath}}-\tilde{\varepsilon}]$ et les voyelles orales $[\mathbf{y}, \boldsymbol{\emptyset}, \mathfrak{x}]$ n'existent pas en arabe. 
Dès lors, et selon toutes les probabilités, le locuteur marocain unilingue et/ou illettré va substituer à ces sons d'emprunt des sons plus proches de son propre système (nous avons déjà dressé la liste de ces sons ci-dessus). Et c'est cette substitution qui a permis l'intégration de tous ces termes dans le système phonologique de l'ADM.

Au niveau des voyelles donc, l'ADM ne reçoit aucune voyelle nouvelle du français et par conséquent il n'y a pas de fertilisation vocalique. Il y a plutôt adaptation de tout un système phonologique étranger au système de langue qui reçoit.

2. Les consonnes

Quant aux consonnes françaises du corpus elles sont parfaitement intégrées et il y a même fertilisation consonantique puisque l'ADM est enrichi de trois consonnes nouvelles inexistantes en $\mathrm{AC}$, à savoir, $\mathrm{P}, \mathrm{V}$ et $\mathrm{t}$ (= le $\mathrm{t}$ de filtre).

a) Le problème du $v$ et du $p$

Le son [v] a souvent été prononcé [f] en ADM, mais il y a quelques années, même des individus illettrés commencent à le prononcer dans des termes d'emprunt. D'ailleurs nous avons obtenu deux prononciations différentes pour manivel $>$ mani$\mathrm{fil} /$ manivil, comme on a tilifizyun/tilivizyun $<$ televizjo - vidyo /fidyo $<$ video, etc.

Il en est de même pour le son [p] qui lui aussi est prononcé de plus en plus dans des termes étrangers, alors qu'il n'y a pas très longtemps il était réalisé surtout [b] en ADM par la majorité des Marocains. Et comme pour le [v] il y a également deux prononciations possibles : [rapel] > rrabbil / rrappil — pižama > e bižama / e ppižama [politik] $>$ e bulitik / e pulitik

b) Le cas du /t/

filtr filtar

le /t/ de filtor n'est ni un $t$ ni un $t$ arabe/ dalyi].

$\mathrm{Ce}[\mathrm{t}]$ a le même timbre que le $1^{\mathrm{er}}[\mathrm{t}]$ français qui existe dans la phrase [tə suvjẽty

Ainsi nos informateurs (comme beaucoup de Marocains) prononcent un [t] français (filtər - tənbər : filtre - timbre) etc., comme des Français. Le [t] français reste donc un [t] français en ADM.

Cependant on peut avoir une $2^{\mathrm{e}}$ prononciation avec un [t] arabe.

D'autre part, pour éviter toute confusion dans la prononciation de ces deux sons j'ai pensé à une notation précise : je propose [ $t]$ pour le $t$ arabe comme le son $[t]$ de [ty] en français et $\left[t_{1}\right]$ pour ce nouveau [ $\left.t\right]$. Enfin je pense donc qu'il y a " fertilisation " phonologique en ce qui concerne le système consonantique de l'ADM. En effet, ces trois phonèmes font partie intégrante du système de l'ADM et permettent des prononciations nouvelles dont on avait grandement besoin.

c) Hypothèse

Cette double prononciation m'a poussé à émettre l'hypothèse suivante : la situation de contact entre les deux codes a créé au niveau consonantique de l'ADM trois nouveaux phonèmes avec deux variantes libres chacun.

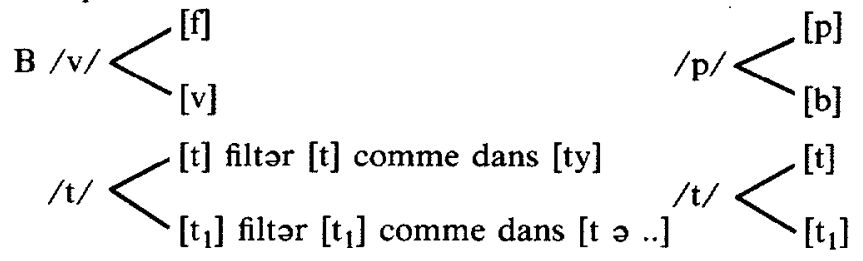




\section{FERTILISATION MORPHOLOGIQUE?}

A. Le problème de genre des noms

Pendant l'enquête j'ai demandé aux informateurs si les noms du corpus sont féminins ou masculins. Je ne suis pas parti de ma propre compétence sur la langue française ; je voulais connaître le sentiment linguistique de mes informateurs sur les termes empruntés.

1. Classement des termes d'après le genre choisi par les informateurs

a) Noms masculins

klamonet - kliyabuži - kliyadwil - kliyagrif - kliyapip - krwaze - lafus - lanklem - lakrik - lleto - lmanifil - lppans - sarūt a krinu — sarut dalfttor — sarüt sippo - ssizay — ttornofis

b) Noms féminins lalim plat - lalim dəmiro - lalim ro - labirit

c) Noms masculins pour les uns, féminins pour les autres [sarut plat - lažuž]

\section{Analyse}

L'analyse ne touchera que les noms qui sont féminins en français mais qui sont jugés masculins par les informateurs, c'est-à-dire tous les noms en (a) moins lakrik et lleto.

Si je dois donner une interprétation au sentiment linguistique des informateurs qui mettent au masculin des termes d'emprunt féminin, je peux trouver une réponse quant aux synapsies qui commencent par le terme [sarūt]. En effet ce terme est du genre masculin en ADM. Ainsi tous les termes sarut (akrinu + dəl filtər + sippo) sont masculins.

Mais pour les autres noms y a-t-il une explication ? Peut-on expliquer le sentiment linguistique des sujets parlants d'une langue donnée?

Tout ce que je peux dire en l'état actuel des recherches c'est que ce phénomène n'est pas un pur hasard, puisqu'il est quasi général. Il doit certainement avoir des causes sous-jacentes assez obscures. C'est pour cette raison qu'il faut se suffire d'une adéquation descriptive pour le moment. Ce que je peux dire c'est que presque tous les Marocains - bilingues (F + ADM) ou non - mettent au masculin la plupart des noms féminins français d'emprunt. On dit bien par exemple : țtẹlevizyūn hmor «(la télévision rouge)".

rradio kbir

" (la radio grand) »

Or si on doit respecter le genre féminin de ces deux noms quand ils passent à l'ADM on dira :

țtelevizyūnhmma

rradio kbira

(La télé est rouge)

(la radio est grande)

Et ce qui est curieux c'est que les bilingues marocains savent que ces deux noms sont féminins en français et continuent à les mettre au masculin quand ils les intégrent dans une phrase ou un discours en ADM.

Les exemples de ce phénomème sont très nombreux dans les termes d'emprunt et c'est pour cette raison - peut-être - que je les ai rencontrés dans le champ lexical du garage. 
B. Le problème du nombre

En ADM on distingue deux pluriels. Le pluriel externe ou "sain " qui se forme par adjonction au nom singulier des suffixes -in, -āt, -a, et le pluriel interne ou «brisé dont les types sont nombreux et variés ${ }^{5}$.

1. Analyse du corpus

a) Corpus

Dans le corpus j'ai rencontré trois types de pluriels. Les deux types que je viens de présenter et un troisième type tout à fait emprunté au français.

Nous classerons le corpus selon cette division.

Termes d'emprunt utilisant le pluriel externe (suffixe -āt) : ce sont les plus nombreux.

$\mathrm{Sg}$ kleamolkt $>$ klamonet

pẽs $>$ ppans

pl. klamonẹtāat

turnavis $>$ tornofis

pl. ppansāt

etc.

pl. tornofisăt

Termes utilisant le pluriel interne

Sg kleplat > sarut plat.

kle si pã > sarut sippo

pl. swarat plat

pl. swarət sippo

Termes dont le pluriel est pris au français

$\begin{array}{ll}\text { français } & \text { ADM } \\ \text { kle a pip } & > \\ \text { kle a dūj } & >\text { lezapip } \\ & \end{array}$

b) Analyse

Je ne traiterai que les $1^{\text {er }}$ et $3^{e}$ groupes de pluriels. En fait le $2^{e}$ groupe forme son pluriel d'une manière régulière.

Mais je donnerai quelques exemples des termes d'emprunt avec le pluriel interne dans des champs autres que celui du garage.

Termes empruntés formant leur pluriel avec -āt

Le suffixe -āt s'adapte bien à un grand nombre de termes du corpus. Et de ce fait il est très rentable.

Les deux autres suffixes (-in, $+-a)$ ne se rencontrent pas dans le corpus. Mais on peut les rencontrer dans d'autres champs lexicaux d'emprunt.

Ex. -in

$$
\begin{aligned}
& \text {-mnərrvi - mnərvin (énervé - énervés) } \\
& \text {-mnofəz - mrofžn (révolté - révoltés) } \\
& \text {-graydi - graydiyya (gardé(s)) } \\
& \text {-žadermi - žadarmiyya (gendarme(s)) }
\end{aligned}
$$

Termes utilisant le pluriel interne

Pour ce pluriel le même terme revient au début de chaque synapsie, à savoir le mot sarūt qui forme son pluriel comme suit : swarat.

Cependant le pluriel interne se rencontre dans d'autres mots, en dehors du champ lexical du garage. 
Termes dont le pluriel est pris au français

ex : lezapid - lezadwil

Je n'ai pas beaucoup de termes de ce type dans le corpus. Mais si ce pluriel se rencontrait dans d'autres champs lexicaux d'emprunt, cela pourrait constituer pour l'arabe marocain un nouveau pluriel.

c) Le problème des verbes

Le corpus n'a pas fourni beaucoup de verbes construits à partir de termes d'emprunt. J'en ai obtenu deux seulement.

tangrisi (graisser) - tangrisi ttomobilāt (je graisse les voitures)

tanrigal (régler) - tanrigal ssoppap (je règle les soupapes)

Mais je peux affirmer sans ambages qu'il existe beaucoup de verbes formés à partir des termes étrangers. Je ne donnerai que quelques exemples car la liste est très longue. Kayvoțēw (voter) - kaydrisi (dresser) - kayksiliri - (accélérer) - tayfrani (freiner) - tay dobel (doubler).

Conclusion sur ce paragraphe

$\mathrm{Y}$ a-t-il fertilisation morphologique de l'ADM par le français ou simple intégration des termes d'emprunt ou système morphologique de l'ADM?

L'intégration est réalisée puisque ces emprunts acceptent le genre, le nombre, etc., sans problèmes.

Cependant, s'il faut distinguer entre intégration et fertilisation, je peux dire qu'il n'y a pas fertilisation morphologique, à part le nouveau pluriel en Lez... (Lezapip). Mais même dans ce cas précis je n'ai pu avoir que quelques termes et il faudra de nombreuses enquêtes dans d'autres champs lexicaux de termes étrangers pour se fixer sur ce nouveau pluriel en lez...

Maintenant s'il n'y a pas de distinction entre fertilisation et intégration, je peux dire qu'il y a fertilisation. En effet le lexique de l'ADM est enrichi de termes nouveaux qui comblent des lacunes considérables dans un des domaines techniques les plus utiles à la vie moderne.

Il y a donc fertilisation au niveau du lexique.

Notes

1. MEILLET (1958) : Linguistique historique et linguistique générale, Paris, Champion.

2. CALVET, L.J. (1974) : Linguistique et colonialisme, Paris, Payot, pp. 60-61.

3. DUBOIS, J. et al. (1973) : Dictionnaire de linguistique, Paris, Larousse, p. 475.

4. Ibid., p. 194.

5. Pour approfondir la question, cf. Ph. MARÇAIS (1977) : Esquisse grammaticale de l'arabe maghrébin, Paris, Librairie d'Amérique et d'Orient, pp. 115-137. 